\title{
Redox Chemistry of Iron in Fog and Stratus Clouds
}

\author{
Yigal ERel ${ }^{1}$, Simo O. Pehronen, AND Michael R. HoffmanN \\ W. M. Keck Laboratories of Environmental Engineering Science, \\ California Institute of Technology. Pasadena
}

\begin{abstract}
The redox chemistry of $\mathrm{Fe}$ in fog and cloudwater has been investigated at coastal and inland locations in the Los Angeles basin, in Bakersfield California, and in Delaware Bay. Samples were collected and analyzed for $\mathrm{Fe}(\mathrm{Fe}(\mathrm{II}), \mathrm{Fe}(\mathrm{III})$, total $\mathrm{Fe}$ ), sulfur (S(IV), S(VI)), organic ligands (formate, acetate, oxalate), total organic carbon (TOC), $\mathbf{p H}$, major cations (sodium, calcium, magnesium, potassium, ammonium), chloride, sulfate, nitrate, peroxides, and aldehydes (HCHO); the amount of sunlight was also measured. The ratio $\mathrm{Fe}(\mathrm{II}) / \mathrm{Fe}(\mathrm{total})$ varied between 0.02 and 0.55 . The concentration of $\mathrm{Fe}$ (II) varied between 0.1 and 5 micromole, and the concentration of total Fe varied between 2 and 27 micromole. The atmospheric redox cycle of Fe involves both dissolved and aerosol surface species and appears to be related to the presence of organic compounds which act as electron donors for the reduction of $\mathrm{Fe}$ (III). $\mathrm{Fe}$ (III) reduction is enhanced by light but significant Fe(II) levels were observed in the dark. We suggest that reduction of $\mathrm{Fe}$ (III) species by organic electron donors may be an important pathway that affects the speciation of Fe in both urban and rural atmospheres. It is possible that reactions involving Fe and organic compounds might be an important source of carboxylic acids in the troposphere.
\end{abstract}

\section{INTRODUCTION}

A knowledge of the exact speciation of all oxidation states of transition metals in atmospheric water droplets as a function of variables such as $p \mathrm{H}$, organic ligand content and ionic strength is critical to the computational assessment of in situ reaction pathways involving S(IV), dissolved oxygen, $\mathrm{RHCO}, \mathrm{NO}_{\mathbf{x}}$, and peroxides [Jacob and Hoffmann, 1983; Hoffmann and Jacob, 1984; Weschler et al., 1986; Faust and Hoigné, 1990]. Likewise, a knowledge of the speciation of metals such as $\mathrm{Fe}$ and $\mathrm{Mn}$ in wet and dry atmospheric deposition and the subsequent speciation changes upon introduction to marine waters is important to the assessment of the ability of marine biota to utilize these atmospherically derived elements for nutritional needs [Jackson and Morgan, 1978; Anderson and Morel, 1982; Duce, 1986; Martin et al., 1988, 1989].

Iron is emitted to the troposphere from both natural (e.g., windblown dust) and anthropogenic (e.g., coal combustion) sources [Morales, 1979; Hansen et al., 1984]. It has been proposed that atmospheric Fe participates in a variety of reactions such as the oxidation of S(IV) and organic compounds by $\mathrm{Fe}$ (III) via direct electron transfer, and the catalytic autooxidation of S(IV) to S(VI) in the droplet phase [Weschler et al., 1986; Pandis and Seinfeld, 1989; Pandis et al., 1992]. In addition, laboratory experiments have shown that Fe(III) is an important oxidant of S(IV) and organic compounds (e.g., aldehydes to carboxylic acids) [Cunningham et al., 1985; Faust and Hoffinann, 1986;

\footnotetext{
1 Also at The Institute of Earth Sciences, The Hebrew University, Jerusalem, Israel 91904.
}

Copyright 1993 by the American Geophysical Union.
Martin et al., 1991]. In spite of the known redox chemistry of $\mathrm{Fe}$, most previous investigations of $\mathrm{Fe}$ in atmospheric water have been limited to the determination of the total dissolved Fe concentration. Moreover, current models of atmospheric cloud chemistry have assumed that $\mathrm{Fe}$ in cloudwater and aerosols is present as mostly Fe(III) in a variety of solid and aqueous phase species [Pandis and Seinfeld, 1989].

Previous measurements of $\mathrm{Fe}(\mathrm{II})$ in seawater and in stream water have shown that it is also present at significant levels in oxic surface water [Hong and Kester, 1986; Landing and Westerlund, 1988; McKnight et al., 1988]. In addition, there are a few recent reports of the presence of $\mathrm{Fe}(\mathrm{II})$ in atmospheric water and aerosols [Behra and Sigg, 1990; Dedik et al., 1992; Zhuang. 1992; Zhuang et al., 1992; Zhu et al., 1993]. A study of the speciation of $\mathrm{Fe}$ in fog collected with the Caltech active strand collector in Zurich [Behra and Sigg, 1990] has shown that a significant fraction of the total Fe (20 to $90 \%$ ) is present as soluble Fe(II) (up to 200 micromole) over a $p \mathrm{H}$ range of 3-7 and that in many samples $\mathrm{Fe}$ (II) was the predominant oxidation state in solution (dissolved $\mathrm{Fe}$ is operationally defined by filtration with either a 0.45 - or 0.05 -micrometer membrane filter).

According to Zhuang et al. [1992] approximately 50\% of the $\mathrm{Fe}$ in aerosols collected from the marine environment is $\mathrm{Fe}(\mathrm{II})$, while only a few percent of the $\mathrm{Fe}$ in aerosols collected over China is Fe(II). Zhuang et al. [1992] attributed the large fraction of $\mathrm{Fe}$ (II) in marine aerosol to multiple cycles of reductive dissolution of ferric oxides followed by oxidation and precipitation taking place during the long journey of these particles in the atmosphere. They also postulated that the redox cycle of Fe is related to that of $S$. Zhu et al. [1993], on the other hand, reported that $\mathrm{Fe}(\mathrm{II})$ makes up only $1 \%$ of the total $\mathrm{Fe}$ and $7.5 \%$ of the soluble $\mathrm{Fe}$ in marine aerosols. Zhu et al. [1993] conducted photolysis experiments with aerosol extracts and with dissolved Fe(III) in aerated acidic sodium chloride solutions both in the pres- 
ence and in the absence of oxalate (as an electron donor). They observed that within a short time, Fe(II) concentration rose from 0.1 micromole to 1.3 micromole (almost $30 \%$ of the total $\mathrm{Fe}$ ) in aerosol extracts exposed to sunlight. The final concentration was attained within less than an hour and was maintained as long as the samples were irradiated. Once the irradiation ceased, the concentrations of $\mathrm{Fe}$ (II) in solution dropped. Based on their experiments with oxalate and dissolved $\mathrm{Fe}$ (III), Zhu et al. [1993] concluded that oxalate participates in the formation of $\mathrm{Fe}$ (II) in irradiated samples via electron transfer within the dissolved Fe-oxalato complexes; however, oxalate oxidation also produces hydrogen peroxide which oxidizes Fe(II) back to Fe(III). The oxidation of $\mathrm{Fe}$ (II) by hydrogen peroxide takes over in the dark, leading to the observed decrease in Fe(II) concentrations. Zhu et al. [1993] did not observe substantial $\mathrm{Fe}$ (II) formation in irradiated goethite suspension in the presence of oxalate.

In light of these considerations we now report on the occurrence of $\mathrm{Fe}$ (II) in stratus clouds collected in coastal and inland environments. In addition, we highlight important processes, chemical species, and critical environmental parameters that affect the dynamic redox cycle of $\mathrm{Fe}$ in the atmosphere.

\section{EXPERIMENTAL METHODS}

To reliably analyze the oxidation states of $\mathrm{Fe}$ and the partitioning of Fe between particulate matter and solution, we have carried out as many measurements as possible in the field. In addition, the low concentrations of Fe expected in some of the samples required that great care be exercised to minimize and monitor blanks during sample collection and handling.

\section{Sample Collection}

Fog and cloudwater samples were collected wilh acidcleaned Caltech active strand cloudwater collectors (CASCC) [Daube et al., 1987]. Concentrations of Fe and other constituents in blanks were determined before each collection. Of all constituents analyzed, Fe blanks were always the highest relative to $\mathrm{Fe}$ concentrations in the samples. Nevertheless, Fe blanks never exceeded $10 \%$ of the lowest Fe value measured in a sample. The collectors were placed in two elevated locations within the Los Angeles basin. These sites were (1) a coastal station on the Palos Verdes peninsula and (2) an inland station at Henninger Flats near Mount Wilson [Munger, 1989]. In addition, we collected samples in Bakersfield California and on board the RV Cape Henlopen (College of Marine Studies, University of Delaware) in Delaware Bay.

\section{Sample-Handling Procedures}

Fog and cloudwater samples were collected over 15 to 120 min intervals. The samples were weighed immediately after collection to determine their volume in order to calculate liquid water content (LWC) [Munger, 1989]. Light intensity was measured with a digital irradiance meter (spectral response from 380 to $750 \mathrm{~nm}$, Biospherical Instruments QSP170B). An aliquot of each sample was removed to determine sample $p \mathrm{H}$. A radiometer PHM80 standard $p \mathrm{H}$ meter with a GK2320C semimicrocombination electrode calibrated with $p \mathrm{H} 4$ and 7 buffers was used to measure the $p \mathrm{H}$ in the field. Aliquots of the collected water samples were processed in real time in the field (including filtration through 0.025 -micrometer pore size Millipore filters). $\mathrm{Fe}$ (II), $\mathrm{Fe}$ (III), and other reactive species were determined by spectrophotometric techniques (see below). The complete determination of reactive species in atmospheric waters other than $\mathrm{Fe}$ (II) and $\mathrm{Fe}$ (III) is beyond the scope of this study. We focused instead on some of the most abundant inorganic and organic compounds in atmospheric water which are likely to exchange electrons with $\mathrm{Fe}$ [Kawamura and Kaplan, 1991; Munger, 1989]. In addition, we determined the total organic carbon (TOC) content as a measure of all the other numerous natural and anthropogenic organic compounds present in atmospheric water. The species that were studied include S(IV)/S(VI), ammonium/nitrate, monocarboxylic acids (e.g, formate, acetate), dicarboxylic acids (oxalate), and formaldehyde. In addition, we monitored changes in the concentration of $\mathrm{Fe}(\mathrm{II}), \mathrm{Fe}$ (III), and $\mathrm{pH}$ as a function of time, in samples stored both in darkness and exposed to daylight.

The aliquots of each sample removed for analysis of reactive species were stabilized in the field. Carboxylic acids (oxalate, acetate, formate) and ammonium were preserved by addition of chloroform [Jacob et al., 1986; Munger, 1989]. Aliquots for TOC analysis were preserved by addition of mercury chloride solution. HCHO (formaldehyde) was reacted with ammonium-acetylacetone to form 3,5-dihydro-1, 4-dihydrolutidine (DDL), which is stable for several weeks [Smith and Erhardt, 1975].

A buffered solution of $p$-hydroxyphenylacetic acid (POPA) and peroxidase was used to preserve peroxides by formation of a fluorescent dimer [Kok et. al., 1986]. Samples for analyses of total concentrations of $\mathrm{Fe}$ and major cations (e.g., sodium, potassium, magnesium, calcium) with a direct current plasma spectrometer (DCP) were acidified (0.1 $\mathrm{M} \mathrm{HCl}$ ) and stored at $4^{\circ} \mathrm{C}$ until analysis. The particulate matter collected on filters was brought back to the laboratory for analysis and was digested with concentrated $\mathrm{HF}$ and $\mathrm{HNO}_{3}$. For several samples, $\mathrm{Fe}_{\mathrm{dgs}}$, the concentration of Fe in filtrate plus filter digest, was compared with Fe concentration in acidified $(\mathrm{pH}=1)$ filtered (Fent) and unfiltered $\left(\mathrm{Fe}_{\mathrm{ac}}\right.$ ) aliquots (all analyzed by a DCP) and with $\mathrm{Fe}(\mathrm{II})$ plus Fe(III) concentration (determined spectrophotometrically) in unfiltered $\left(\mathrm{Fe}_{\text {reac }}\right)$ and filtered aliquots.

\section{Spectrophotometric Methods of Analyses}

Spectrophotometric analyses were performed with (1) Shimadzu UV-1201 portable spectrophotometer with a 5cm cell, (2) Shimadzu RF-540 recording spectrofluorophotometer, and (3) Shimadzu 500 DOC/TOC analyzer (100 ppb detection limit).

Iron. For $\mathrm{Fe}$ (II) determination we applied two methods: (1) the ferrozine (3-(2-pyridyl)-5,6-diphenyl-1,2,4-triazinep,p'-disulfonic acid, monosodium salt monohydrate) method used originally by Stookey [1970] and Carter [1971] and which is the most common method for Fe(II) determination in water and blood serum samples; (2) Di-2-pyridyl ketone benzoylhydrazone (DPKBH) was selected as a second chelating agent for $\mathrm{Fe}$, because it is known to complex both $\mathrm{Fe}(\mathrm{II})$ and $\mathrm{Fe}$ (III) and because of its low solubility in wates which enables a preconcentration step [Garcia-Vargas et al., 1986; Zatar et al., 1989; Pehkonen et al., 1992]. 
Iron chelation by DPKBH both with and without a preconcentration procedure was carried out in order to cover a wide range of $\mathrm{Fe}$ concentrations. A spectrophotometric detection limit of 4 nanomole for both Fe(III) and Fe(II) with a linear response from 4 nanomole up to 0.1 micromole was established for samples extracted with chloroform-water. DPKBH chelation without chloroform extraction showed a linear response from 0.1 micromole to 30 micromole. The molar extinction coefficients of the bis-Fe(II)DPKBH and bis-Fe(III)-DPKBH complexes are given in Pehkonen et al. [1992]. Analytical interference studies on the possible changes in the oxidation state of $\mathrm{Fe}$ with S(IV), oxalate, and other potential electron donors have also been carried out [Pehkonen et al., 1992].

Spectrophotometric measurements of other compounds. HCHO was measured after addition of iodine (light absorption at $415 \mathrm{~nm}$ ) [Smith et al., 1975]. S(IV) was analyzed by the "Bunte salt" method (light absorption at $412 \mathrm{~nm}$ ) [Humphrey et. al., 1970]. Peroxide was measured by the fluorescence of the POPA dimer (peroxide excitation at 320 $\mathrm{nm}$ and emission at $406 \mathrm{~nm}$ ) [Kok et. al., 1986]. This method is sensitive to both hydrogen peroxide and some organic peroxides.

\section{Other Chemical Analyses}

Ion chromatography.Chloride, nitrate, sulfate, acetate, formate, oxalate, and ammonium were measured in the laboratory using a Dionex BIOLC ion chromatograph with PAX-500 anion column and a $\mathrm{NaOH}$ eluent. Gradient elution was employed to enhance the separation of the weakly retained acids and shortening the retention of the unwanted carbonate peaks. One millimole $\mathrm{NaOH}$ solution was mixed with 200 millimole $\mathrm{NaOH}$ solution at different proportions to gradually increase concentration of $\mathrm{NaOH}$ and therefore to improve the elution of ions such as sulfate and oxalate.

Atomic emission and absorption techniques. sodium, potassium, calcium, magnesium, and iron were analyzed with an ARL SpectroSpan VB direct current plasma (DCP) spectrometer. The lower linearity ranges of the instrument used were $2.6,4.1,1.3,0.4,0.9$ micromole, respectively. The coefficients of variance (CV) of calion analysis by the DCP were, at most, $10 \%$. Samples with low iron concentrations were analyzed with a 3030 Perkin Elmer graphite furnace atomic absorption spectrometer (detection limit, 0.02 micromole and a coefficient of variance, $20 \%$ ).

\section{Computational Determination of Chemical Speciation}

With the aid of SURFEQL [Morel and Morgan, 1972; Faughnan, 1981; Jacob and Hoffmann, 1983] we used the equilibrium constant approach to solve a complex chemical equilibrium problem, which is defined by a system of mass action equations [Westall, 1987]. The thermodynamic data base consists of equilibrium constants for more than 1500 equilibria. This data base was verified and supplemented for the present calculation.

\section{RESULTS AND DISCUSSION}

A wide range of concentrations of measured constituents were observed in the collected fog and cloudwater samples (Table 1). Inorganic anions (chloride, nitrate, and sulfate) and cations (ammonium, sodium, and proton) were the dominant species in cloudwater with their concentrations approaching millimole values. The $p \mathrm{H}$ varied between 2.2 and 7.1. The concentration of soluble Fe(II) in cloudwater ranged from 0.1 to 5.3 micromole, while the concentration of Feac varied between 1.8 and 27 micromole(Table 1). The concentrations of peroxides, S(IV), formate, acetate, oxalate, and $\mathrm{HCHO}$ were found to be of the same order of magnitude as the Feac (Table 1).

To test the role of light in controlling the reduction of $\mathrm{Fe}(\mathrm{III})$, we monitored the production of $\mathrm{Fe}$ (II) as function of time both in the light (approximately $5 \times 1016$ quanta/s $\mathrm{cm}^{2}$ of sunlight) and in the dark immediately after the collection of cloudwater samples which contained appreciable quantities of S(IV) and organic compounds (Table 2). Fe(II) concentrations always increased faster in the presence of light than in the dark. Nevertheless, the observed coexistence of Fe(II) and hydrogen peroxide (which itself is a major oxidant of $\mathrm{Fe}(\mathrm{II})$ at the $\mathrm{pH}$ range of most of the cloudwater samples [Martin et al., 1991]) in cloudwater samples that were collected in the dark both in California and in Delaware (Table 1) suggests a thermal reduction pathway of $\mathrm{Fe}$ (III). In addition, Fe(II) values in cloudwater samples collected during the night in Los Angeles were comparable to those found in samples collected long after sunrise (Figure 1a). It has been shown previously that thermal reduction of $\mathrm{Fe}(\mathrm{III})$ by organic compounds that are present in cloudwater (e.g., phenols, aldehydes but not acetate and formate) and by $S(I V)$ is much slower and much less important than photoreduction [Cunningham et al., 1985; Faust and Hoffmann, 1986. Thus our measurements suggest that either thermal reduction is a viable complementary pathway for the in situ production of $\mathrm{Fe}$ (II) in the atmosphere (in contrast to laboratory expcriments) or that both reduction and oxidation rates decrease during nighttime to the same extent, leading to unchanged steady state levels of $\mathrm{Fe}(\mathrm{II})$.

We found a strong linear correlation between the concentrations of $\mathrm{Fe}$ of both oxidation states (Fe(III), $\mathrm{Fe}(\mathrm{II})$ ) and the concentrations of both oxidation states of $S$ (S(IV), $\mathrm{S}(\mathrm{VI})$ ) (Figures $1 b$ and $1 c$ ). This type of correlation indicates that the relationship between Fe(II) and S(IV) is not determined solely by in situ redox chemistry but that their relationship might also be controlled by a common source of $S$ and $\mathrm{Fe}$. The measured values of $\mathrm{S} / \mathrm{Cl}$ ratios suggest that most of the $S$ in the cloudwater samples comes from nonmarine (most likely anthropogenic) sources. More study is needed to test the importance of $\mathrm{Fe}$ in controlling the oxidation of S(IV) in cloud and fog samples, as well as the possibility that the reversible formation of aldehydebisulfite adducts prevent S(IV) from complexing $\mathrm{Fe}$ in cloud and fog samples [Conklin and Hoffmann, 1988; $\mathrm{Ol}$ son and Hoffmann, 1989].

Among the organic compounds in cloudwater, we studied formaldehyde, formate, acetate, and oxalate as potential electron donors for the reduction of Fe(III) to Fe(II) [Munger, 1989; Kawamura and Kaplan, 1991]. The sum of formaldehyde concentration plus acetate plus formate in the cloudwater samples correlates strongly with TOC (R2 = 0.984 ) and comprises approximately $20 \%$ of the TOC (oxalate values were below detection limit in many of the samples). A plot of $[\mathrm{Fe}(\mathrm{II})]$ versus formate in the Los Angeles samples shows two distinctive trends (Figure $2 a$ ). The data points that are correlated along the line with a steeper slope (i.e., more Fe(II) per mole of formate) represent cloudwater samples that have higher total $\mathrm{Fe}\left(\mathrm{Fe}_{\mathrm{ac}}\right)$ concentrations (Figure $2 b$ ) and, in general, higher ac- 
TABLE 1. The Concentrations of Various Components Measured in Fog and Cloudwater Samples

\begin{tabular}{|c|c|c|c|c|c|c|c|c|}
\hline No. & $\begin{array}{c}\text { Site } \\
\text { Location }\end{array}$ & $\begin{array}{c}\text { Collection } \\
\text { Date }\end{array}$ & $\begin{array}{c}\text { Sample } \\
\text { Description }\end{array}$ & $\begin{array}{l}\text { Collection } \\
\text { Time }\end{array}$ & $\begin{array}{l}\text { Duration, } \\
\text { min }\end{array}$ & $\begin{array}{c}\text { Weight, } \\
\text { g }\end{array}$ & $\begin{array}{l}\mathrm{LWC}, \\
\mathrm{g} / \mathrm{m}^{3}\end{array}$ & pH \\
\hline 1 & Henninger & $4 / 25 / 91$ & unfiltered & 0215 & 120 & NA & NA & 3.1 \\
\hline 2 & Henninger & $5 / 30 / 91$ & filtered collector 1 & 0130 & 45 & 100 & 0.11 & NA \\
\hline 3 & Henninger & $5 / 30 / 91$ & filtered collector 3 & 0130 & 45 & 100 & 0.11 & NA \\
\hline 4 & Henninger & $5 / 30 / 91$ & unfiltered collector 1 & 0130 & 45 & 100 & 0.11 & NA \\
\hline 5 & Henninger & $5 / 30 / 91$ & unfiltered collector 3 & 0130 & 45 & 100 & 0.11 & NA \\
\hline 6 & Henninger & $5 / 30 / 91$ & filter digest collector 3 & 0130 & 45 & 100 & 0.11 & NA \\
\hline 7 & Henninger & $5 / 30 / 91$ & filtered collector 1 & 0215 & 45 & 100 & 0.11 & 2.5 \\
\hline 8 & Henninger & $5 / 30 / 91$ & filtered collector 3 & 0215 & 45 & 100 & 0.11 & 2.5 \\
\hline 9 & Henninger & $5 / 30 / 91$ & unfiltered collector 1 & 0215 & 45 & 100 & 0.11 & 2.5 \\
\hline 10 & Henninger & $5 / 30 / 91$ & unfiltered collector 3 & 0215 & 45 & 100 & 0.11 & 2.5 \\
\hline 11 & Henninger & $5 / 30 / 91$ & filter digest collector 3 & 0215 & 45 & 100 & 0.11 & 2.5 \\
\hline 12 & Henninger & $5 / 30 / 91$ & filter digest collector 3 & 0215 & 45 & 100 & 0.11 & 2.5 \\
\hline 13 & Henninger & $5 / 30 / 91$ & filtered collector 1 & 0300 & 45 & 100 & 0.11 & 2.8 \\
\hline 14 & Henninger & $5 / 30 / 91$ & filtered collector 3 & 0300 & 45 & 100 & 0.11 & 2.8 \\
\hline 15 & Henninger & $5 / 30 / 91$ & unfiltered collector 1 & 0300 & 45 & 100 & 0.11 & 2.8 \\
\hline 16 & Henninger & $5 / 30 / 91$ & unfiltered collector 3 & 0300 & 45 & 100 & 0.11 & 2.8 \\
\hline 17 & Henninger & $5 / 30 / 91$ & filtered collector 1 & 0345 & 75 & 220 & 0.14 & 2.8 \\
\hline 18 & Henninger & $5 / 30 / 91$ & filtered collector 3 & 0345 & 75 & 220 & 0.14 & 2.8 \\
\hline 19 & Henninger & $5 / 30 / 91$ & unfiltered collector 1 & 0345 & 75 & 220 & 0.14 & 2.8 \\
\hline 20 & Henninger & $5 / 30 / 91$ & unfiltered collector 3 & 0345 & 75 & 220 & 0.14 & 2.8 \\
\hline 21 & Henninger & $5 / 30 / 91$ & filter digest collector 3 & 0345 & 75 & 220 & 0.14 & 2.8 \\
\hline 22 & San Pedro & $6 / 11 / 91$ & unfiltered & 0750 & 20 & 30 & 0.07 & 4.2 \\
\hline 23 & San Pedro & $6 / 11 / 91$ & unfiltered & 0825 & 25 & 28 & 0.05 & 4.0 \\
\hline 24 & San Pedio & $6 / 11 / 91$ & unfiltered & 0900 & 25 & 61 & 0.11 & 3.8 \\
\hline 25 & San Pedro & $6 / 11 / 91$ & unfilltered & 0930 & 25 & 57 & 0.11 & 3.8 \\
\hline 26 & San Pedro & $6 / 11 / 91$ & unfiltered & 1000 & 30 & 48 & 0.08 & 3.5 \\
\hline 27 & San Pedro & $6 / 11 / 91$ & unfiltered & 1035 & 15 & 21 & 0.07 & NA \\
\hline 28 & Henninger & $6 / 27 / 91$ & unfiltered & 0845 & 35 & 65 & 0.09 & 3.9 \\
\hline 29 & Henṇinger & $6 / 27 / 91$ & filtered & 0845 & 35 & 65 & 0.09 & 3.9 \\
\hline 30 & Henninger & $6 / 27 / 91$ & filter digest & 0845 & 35 & 65 & 0.09 & 3.9 \\
\hline 31 & Henninger & $6 / 27 / 91$ & filter digest & 0845 & 35 & 65 & 0.09 & 3.9 \\
\hline 32 & Henninger & $6 / 27 / 91$ & unfiltered & 0920 & 30 & 25 & 0.04 & 3.7 \\
\hline 33 & San Pedro & $7 / 10 / 91$ & unfiltered & 1000 & 35 & 30 & 0.04 & 3.4 \\
\hline 34 & San Pedro & $7 / 23 / 91$ & unfilltered & 0855 & 25 & 95 & 0.18 & 3.2 \\
\hline 35 & San Pedro & $7 / 23 / 91$ & unfiltered & 0920 & 20 & 90 & 0.21 & 2.5 \\
\hline 36 & San Pedro & $7 / 23 / 91$ & unfiltered & 0950 & 15 & 54 & 0.17 & 2.2 \\
\hline 37 & San Pedro & $7 / 23 / 91$ & unfiltered & 1020 & 25 & 60 & 0.11 & 2.4 \\
\hline 38 & San Pedro & $7 / 23 / 91$ & unfiltered & 1050 & 25 & 30 & 0.06 & 2.8 \\
\hline 39 & San Pedro & $7 / 23 / 91$ & unfiltered & 1125 & 45 & 23 & 0.02 & 2.6 \\
\hline 40 & San Pedro & $7 / 25 / 91$ & unfiltered & 0830 & 35 & 60 & 0.08 & 3.2 \\
\hline 41 & San Pedro & $7 / 25 / 91$ & filtered & 0830 & 35 & 60 & 0.08 & 3.2 \\
\hline 42 & San Pedro & $7 / 25 / 91$ & filter digest & 0830 & 35 & 60 & 0.08 & 3.2 \\
\hline 43 & San Pedro & $7 / 25 / 91$ & filter digẹst & 0830 & 35 & 60 & 0.08 & 3.2 \\
\hline 44 & San Pedro & $7 / 25 / 91$ & unfiltered & 0905 & 50 & 65 & 0.06 & 2.6 \\
\hline 45 & San Pedro & $7 / 25 / 91$ & filtered & 0905 & 50 & 65 & 0.06 & 2.6 \\
\hline 46 & San Pedro & $7 / 25 / 91$ & filter digest & 0905 & 50 & 65 & 0.06 & 2.6 \\
\hline 47 & San Pedro & $7 / 25 / 91$ & unfiltered & 0955 & 40 & 33 & 0.04 & 3.4 \\
\hline 48 & Delaware & $3 / 5 / 92$ & unfiltered & 0515 & 30 & 15 & 0.02 & NA \\
\hline 49 & Delaware & $3 / 5 / 92$ & unfiltered & 0545 & 15 & 31 & 0.10 & 2.9 \\
\hline 50 & Delaware & $3 / 5 / 92$ & unfiltered & 0605 & 15 & 6 & 0.02 & NA \\
\hline 51 & Delaware & $3 / 5 / 92$ & unfiltered & 0705 & 115 & 91 & 0.04 & 3.8 \\
\hline 52 & San Pedro & $6 / 5 / 92$ & unfiltered & 1047 & 18 & 48 & 0.13 & 4.4 \\
\hline 53 & San Pedro & $6 / 5 / 92$ & unfiltered & 1105 & 30 & 30 & 0.05 & 4.3 \\
\hline 54 & San Pedro & $6 / 5 / 92$ & unfiltered & 1135 & 25 & 8 & 0.01 & 4.2 \\
\hline 55 & Bakersfield & $1 / 27 / 93$ & unfiltered & 1125 & 55 & 68 & 0.06 & 6.4 \\
\hline 56 & Bakersfield & $1 / 28 / 93$ & unfiltered & 1225 & 42 & 131 & 0.15 & 6.7 \\
\hline 57 & Bakersfield & $1 / 28 / 93$ & unfiltered & 0107 & 46 & 123 & 0.13 & 6.8 \\
\hline 58 & Bakersfield & $1 / 28 / 93$ & unfiltered & 0153 & 53 & 127 & 0.11 & 7.1 \\
\hline 59 & Bakersfield & $1 / 28 / 93$ & unfiltered & 0246 & 60 & 129 & 0.10 & 6.8 \\
\hline
\end{tabular}


TABLE 1. (continued)

Light, Temp, $\mathrm{Fe}(\mathrm{II})^{*} \mathrm{Fe}(\mathrm{III})^{*} \quad \mathrm{Fe}(\mathrm{II})^{*} \quad \mathrm{Fe}$

No. $q /(\mathrm{s} \mathrm{cm} 2)$ c (DPKBH) (DPKBH) (Ferrozine) (DCP) Peroxide Oxalate HCHO Formate Acetate

\begin{tabular}{|c|c|c|c|c|c|c|c|c|c|c|c|}
\hline 1 & dark & NA & 13.1 & 5.0 & NA & NA & 12.0 & BDL & 3.6 & 55.4 & BDL \\
\hline 2 & dark & NA & $\mathrm{NA}$ & NA & NA & NA & 4.2 & BDL & 41 & 56.3 & 19.3 \\
\hline 3 & dark & NA & NA & NA & NA & 9.1 & 4.2 & BDL & 41 & 56.3 & 19.3 \\
\hline 4 & dark & NA & NA & NA & NA & 16.9 & 4.2 & BDL & 41 & 56.3 & 19.3 \\
\hline 5 & dark & $\mathrm{NA}$ & NA & NA & NA & 25.5 & 4.2 & BDL & 41 & 56.3 & 19.3 \\
\hline 6 & dark & 11.0 & NA & NA & NA & 25.9 & NA & NA & NA & NA & NA \\
\hline 7 & dark & 11.0 & NA & NA & NA & NA & 4.6 & BDL & 35 & 56.9 & 26.3 \\
\hline 8 & dark & 11.0 & NA & NA & NA & 4.0 & 4.6 & BDL & 35 & 56.9 & 26.3 \\
\hline 9 & dark & 11.0 & 4.9 & 6.7 & NA & 26.5 & 4.6 & BDL & 35 & 56.9 & 26.3 \\
\hline 10 & dark & 11.0 & 2.9 & 6.1 & NA & 20.7 & 4.6 & BDL & 35 & 56.9 & 26.3 \\
\hline 11 & dark & 11.0 & NA & NA & NA & 19.0 & NA & NA & NA & $\mathrm{NA}$ & NA \\
\hline 12 & dark & 11.0 & NA & NA & NA & 17.6 & NA & NA & NA & NA & NA \\
\hline 13 & dark & 10.5 & NA & NA & NA & NA & 5.2 & BDL & 48 & 46.2 & BDL \\
\hline 14 & dark & 10.5 & NA & NA & NA & NA & 5.2 & BDL & 48 & 46.2 & BDL \\
\hline 15 & dark & 10.5 & 4.1 & 3.3 & NA & 13.2 & 5.2 & BDL & 48 & 46.2 & BDL \\
\hline 16 & dark & 10.5 & 3.8 & 4.1 & $\mathrm{NA}$ & 10.9 & 5.2 & BDL & 48 & 46.2 & BDL \\
\hline 17 & dark & 10.5 & NA & NA & $\mathrm{NA}$ & NA & 3.5 & BDL & 47 & 54.6 & 11.4 \\
\hline 18 & dark & 10.5 & 0.0 & 2.3 & NA & 2.2 & 3.5 & BDL & 47 & 54.6 & 11.4 \\
\hline 19 & dark & 10.5 & 1.2 & 2.0 & NA & 4.3 & 3.5 & BDL & 47 & 54.6 & 11.4 \\
\hline 20 & dark & 10.5 & 0.9 & 2.5 & NA & 6.7 & 3.5 & BDL & 47 & 54.6 & 11.4 \\
\hline 21 & dark & 10.5 & NA & NA & NA & 23.7 & NA & NA & NA & NA & NA \\
\hline 22 & $3.3 E+16$ & NA & NA & NA & $\mathrm{NA}$ & NA & 33.7 & BDL & 19.4 & 29.9 & BDL \\
\hline 23 & $5.2 \mathrm{E}+16$ & NA & 3.9 & 4.2 & $\mathrm{NA}$ & 4.5 & 48.1 & BDL & 16.8 & 43.4 & BDL \\
\hline 24 & $5.4 E+16$ & NA & 1.4 & 1.2 & NA & 2.6 & 29.4 & BDL & 17.8 & 26.2 & BDL \\
\hline 25 & $6.3 E+16$ & NA & 1.5 & 1.1 & NA & 2.3 & 53.4 & BDL & 17.9 & 30.1 & BDL \\
\hline 26 & $7.6 \mathrm{E}+16$ & NA & 2.0 & 1.5 & NA & 2.9 & 64.2 & BDL & 17.4 & 47.5 & $\mathrm{BDL}$ \\
\hline 27 & $9.0 \mathrm{E}+16$ & NA & 4.3 & 3.2 & NA & 3.3 & 78.6 & BDL & 19.7 & 51.3 & BDL \\
\hline 28 & $1.0 \mathrm{E}+16$ & 16.0 & 7.6 & 3.4 & 4.2 & 8.2 & 2.1 & BDL & 23 & 26.5 & BDL \\
\hline 29 & $1.0 \mathrm{E}+16$ & 16.0 & NA & NA & NA & 7.0 & 2.1 & BDL & 23 & 26.5 & BDL \\
\hline 30 & $1.0 \mathrm{E}+16$ & 16.0 & NA & NA & NA & 1.5 & NA & $\mathbf{N A}$ & NA & NA & NA \\
\hline 31 & $1.0 \mathrm{E}+16$ & 16.0 & NA & NA & NA & 1.5 & NA & NA & NA & $\mathrm{NA}$ & NA \\
\hline 32 & $7.5 E+16$ & 16.0 & 7.7 & 2.4 & 5.3 & 9.7 & 1.5 & BDL & 25.6 & 28.6 & BDL \\
\hline 33 & $6.0 \mathrm{E}+16$ & 18.0 & 1.1 & 0.0 & 1.1 & 3.9 & 7.0 & BDL & 32.1 & 23.6 & 36.6 \\
\hline 34 & $3.2 E+16$ & 15.0 & 7.9 & 4.1 & 3.8 & 9.5 & 3.6 & BDL & 12 & 28.1 & 24.9 \\
\hline 35 & $3.7 \mathrm{E}+16$ & 15.0 & 8.0 & 3.3 & 4.7 & 10.2 & 4.8 & BDL & 12.2 & 27.2 & 26.6 \\
\hline 36 & $4.0 \mathrm{E}+16$ & 15.0 & 9.9 & $\mathrm{NA}$ & $\mathrm{NA}$ & 9.0 & 4.8 & BDL & 11.7 & 29.6 & 32.0 \\
\hline 37 & $5.0 \mathrm{E}+16$ & 15.5 & 9.3 & NA & NA & 8.8 & 8.3 & $\mathrm{BDL}$ & 11.5 & 34.3 & 36.9 \\
\hline 38 & $6.4 \mathrm{E}+16$ & 16.2 & 11.1 & NA & NA & 12.2 & 9.6 & 5.7 & 12.3 & 42.6 & 47.6 \\
\hline 39 & $9.5 E+16$ & 19.0 & 18.7 & NA & NA & 20.6 & 6.2 & 9.1 & 15.7 & 75.6 & 88.2 \\
\hline 40 & $1.7 \mathrm{E}+16$ & 14.5 & 1.7 & 1.2 & 0.5 & 2.0 & 24.3 & 7.7 & 8.62 & 22.7 & 17.4 \\
\hline 41 & $1.7 E+16$ & 14.5 & 0.7 & 0.3 & NA & 1.4 & 24.3 & 7.7 & 8.62 & 22.7 & 17.4 \\
\hline 42 & $1.7 \mathrm{E}+16$ & 14.5 & NA & NA & NA & 4.4 & NA & NA & NA & NA & NA \\
\hline 43 & $1.7 \mathrm{E}+16$ & 14.5 & NA & $\mathrm{NA}$ & NA & 4.2 & NA & NA & NA & NA & NA \\
\hline 44 & $3.0 \mathrm{E}+16$ & 14.5 & 1.1 & 0.8 & 0.3 & 1.8 & 32.6 & 8.3 & 8.35 & 27.7 & 21.7 \\
\hline 45 & $3.0 \mathrm{E}+16$ & 14.5 & 0.4 & 0.1 & NA & BDL & 32.6 & 8.3 & 8.35 & 27.7 & 21.7 \\
\hline 46 & $3.0 \mathrm{E}+16$ & 14.5 & NA & NA & NA & 4.3 & NA & NA & NA & NA & NA \\
\hline 47 & $4.0 \mathrm{E}+16$ & 14.5 & 1.8 & 1.2 & 0.7 & 2.7 & 40.5 & 13.2 & 10.1 & 39.8 & 36.8 \\
\hline 48 & $1.1 \mathrm{E}+15$ & NA & NA & NA & NA & 4.2 & NA & NA & NA & NA & $N A$ \\
\hline 49 & $4.2 E+14$ & NA & NA & NA & 0.7 & 3.0 & NA & 16.8 & NA & 21.2 & 49.0 \\
\hline 50 & $7.0 \mathrm{E}+13$ & NA & NA & NA & NA & NA & NA & 13.5 & NA & 13.4 & 25.6 \\
\hline 51 & $2.0 \mathrm{E}+13$ & NA & NA & NA & 0.4 & 1.0 & NA & 8.6 & NA & 16.9 & 19.4 \\
\hline 52 & $7.4 \mathrm{E}+16$ & 14.0 & 0.6 & 0.6 & 0.6 & 0.9 & NA & 13.5 & 1.52 & 31.7 & 30.5 \\
\hline 53 & $8.5 E+16$ & 14.0 & 1.5 & 1.2 & 1.1 & 2.0 & $\mathrm{NA}$ & 1.5 & $\mathrm{NA}$ & 14.3 & 10.7 \\
\hline 54 & $1.0 \mathrm{E}+17$ & 14.0 & 2.0 & 1.6 & NA & NA & NA & 5.3 & NA & 19.5 & 18.2 \\
\hline 55 & dark & 10.0 & NA & NA & 0.1 & 5.9 & 0.0 & 8.5 & NA & 66.0 & 153.0 \\
\hline 56 & dark & 10.0 & NA & NA & 0.3 & 5.4 & 1.1 & 4.0 & NA & 40.0 & 92.0 \\
\hline 57 & dark & 10.0 & NA & NA & 0.2 & 2.7 & 1.4 & 5.0 & NA & 45.0 & 109.0 \\
\hline 58 & dark & 10.0 & NA & NA & 0.3 & 4.9 & 2.4 & 4.0 & NA & 38.0 & 119.0 \\
\hline 59 & dark & 10.0 & NA & NA & 0.2 & 2.8 & 5.4 & 4.0 & NA & 61.0 & 123.0 \\
\hline
\end{tabular}


TABLE 1. (continued)

\begin{tabular}{|c|c|c|c|c|c|c|c|c|c|c|}
\hline No. & $\begin{array}{l}\mathrm{TOC} \\
\mathrm{mg} / 1\end{array}$ & $S(I V)$ & Sulfate & $\mathrm{Cl}$ & Nitrate & Ammonium & $\mathrm{Na}$ & $\mathbf{K}$ & $\mathrm{Ca}$ & $\mathbf{M g}$ \\
\hline 1 & NA & NA & 376 & 2299 & 2563 & 1400 & 960 & 49 & 38 & $\mathbf{N A}$ \\
\hline 2 & NA & NA & 631 & 947 & 3342 & 1500 & $\mathrm{NA}$ & NA & $\mathbf{N A}$ & NA \\
\hline 3 & NA & NA & 631 & 947 & 3342 & 1500 & 481 & 20 & 65 & 60 \\
\hline 4 & NA & NA & 631 & 947 & 3342 & 1500 & 343 & 17 & 45 & 45 \\
\hline 5 & NA & NA & 631 & 947 & 3342 & 1500 & 493 & 25 & 63 & 64 \\
\hline 6 & NA & NA & NA & $\mathrm{NA}$ & NA & NA & 19 & 16 & 5 & 14 \\
\hline 7 & NA & NA & 320 & 589 & 1639 & 1300 & NA & NA & NA & $\mathbf{N A}$ \\
\hline 8 & NA & NA & 320 & 589 & 1639 & 1300 & 552 & 20 & 58 & 65 \\
\hline 9 & NA & NA & 320 & 589 & 1639 & 1300 & 627 & 32 & 64 & 78 \\
\hline 10 & NA & NA & 320 & 589 & 1639 & 1300 & 567 & 25 & 54 & 69 \\
\hline 11 & NA & $\mathrm{NA}$ & NA & $\mathrm{NA}$ & $\mathrm{NA}$ & $\mathrm{NA}$ & BDL & 11 & 6 & 8 \\
\hline 12 & NA & NA & NA & $\mathrm{NA}$ & NA & NA & $\overline{\mathrm{BDL}}$ & 12 & 5 & 6 \\
\hline 13 & NA & NA & 382 & 595 & 1989 & 1400 & NA & $\mathrm{NA}$ & NA & NA \\
\hline 14 & $\mathrm{NA}$ & NA & 382 & 595 & 1989 & 1400 & NA & $\mathrm{NA}$ & $\mathrm{NA}$ & $\mathrm{NA}$ \\
\hline 15 & NA & $\mathrm{NA}$ & 382 & 595 & 1989 & 1400 & 345 & 16 & 27 & 42 \\
\hline 16 & $\mathrm{NA}$ & NA & 382 & 595 & 1989 & 1400 & 307 & 14 & 26 & 41 \\
\hline 17 & $\mathrm{NA}$ & NA & 313 & 539 & 1777 & 1100 & NA & $\mathrm{NA}$ & NA & NA \\
\hline 18 & NA & NA & 313 & 539 & 1777 & 1100 & 213 & 9 & 16 & 26 \\
\hline 19 & NA & NA & 313 & 539 & 1777 & 1100 & 224 & 10 & 18 & 28 \\
\hline 20 & NA & NA & 313 & 539 & 1777 & 1100 & 222 & 9 & 17 & 30 \\
\hline 21 & NA & $\mathrm{NA}$ & NA & $\mathrm{NA}$ & NA & NA & 19 & 16 & 6 & 15 \\
\hline 22 & 19.5 & 17.8 & 397 & 444 & 757 & $\mathrm{NA}$ & $\mathrm{NA}$ & $\mathrm{NA}$ & NA & $\mathrm{NA}$ \\
\hline 23 & 11.3 & 2.4 & 185 & 206 & 426 & $\mathrm{NA}$ & 16 & 4 & 5 & 6 \\
\hline 24 & 11.4 & 0.0 & 206 & 636 & 323 & $\mathrm{NA}$ & 22 & 4 & 7 & 7 \\
\hline 25 & 8.4 & 0.0 & 193 & 702 & 359 & NA & 31 & 4 & 7 & 7 \\
\hline 26 & 11.1 & 1.9 & 239 & 729 & 461 & $\mathrm{NA}$ & 60 & 7 & 17 & 12 \\
\hline 27 & 16.1 & 0.9 & 318 & 931 & 602 & NA & 106 & 10 & 30 & 19 \\
\hline 28 & 14.0 & 1.9 & 64 & 2320 & 392 & 630 & 199 & 10 & 24 & 27 \\
\hline 29 & NA & 1.9 & 64 & 2320 & 392 & 630 & 193 & 9 & 22 & 26 \\
\hline 30 & NA & $\mathrm{NA}$ & NA & $\mathrm{NA}$ & NA & NA & BDL & 1 & 2 & BDL \\
\hline 31 & NA & NA & NA & NA & $\mathrm{NA}$ & $\mathrm{NA}$ & $\mathrm{BDL}$ & 1 & 2 & BDL \\
\hline 32 & NA & 2.4 & 61 & 2700 & 679 & 700 & 352 & 17 & 41 & 46 \\
\hline 33 & 15.0 & 7.7 & 364 & 118 & 572 & 880 & 31 & 8 & 29 & 8 \\
\hline 34 & 10.4 & 17.0 & 411 & 163 & 839 & 730 & 232 & 12 & 46 & 35 \\
\hline 35 & 10.8 & 15.0 & 394 & 168 & 869 & 690 & 223 & 13 & 59 & 38 \\
\hline 36 & 13.5 & 13.0 & 503 & 179 & 1040 & 790 & 250 & 12 & 69 & 43 \\
\hline 37 & 14.8 & 12.7 & 644 & 238 & 1180 & 1000 & 337 & 16 & 85 & 55 \\
\hline 38 & $\mathrm{NA}$ & 15.3 & 903 & 315 & 1550 & 1200 & 441 & 16 & 160 & 74 \\
\hline 39 & $\mathrm{NA}$ & 22.1 & 1400 & 470 & 2480 & $\mathrm{NA}$ & 721 & 39 & 260 & 133 \\
\hline 40 & 7.1 & 7.1 & 147 & 56 & 284 & 440 & 29 & 6 & 13 & 6 \\
\hline $4 ?$ & 7.1 & 7.1 & 147 & 56 & 284 & 440 & 28 & 6 & 13 & 5 \\
\hline 42 & NA & NA & $\mathrm{NA}$ & NA & $\mathrm{NA}$ & $\mathrm{NA}$ & BDL & 4 & 5 & $\mathrm{BDL}$ \\
\hline 43 & NA & NA & NA & NA & $\mathrm{NA}$ & $\mathrm{NA}$ & $\overline{\mathrm{BDL}}$ & 4 & 5 & $\mathrm{BDL}$ \\
\hline 44 & 7.4 & 5.8 & 167 & 62 & 304 & 560 & 20 & 6 & BDL & 4 \\
\hline 45 & 7.4 & 5.8 & 167 & 62 & 304 & 560 & 18 & 5 & BDL & 4 \\
\hline 46 & $\mathrm{NA}$ & NA & NA & $\mathrm{NA}$ & $\mathrm{NA}$ & $\mathrm{NA}$ & BDL & 4 & 5 & BDL \\
\hline 47 & 12.3 & 4.2 & 264 & 95 & 515 & 1500 & 26 & 9 & 22 & 9 \\
\hline 48 & $\mathrm{NA}$ & $\mathrm{NA}$ & $\mathrm{NA}$ & NA & $\mathrm{NA}$ & $\mathrm{NA}$ & 91 & 25 & 79 & 70 \\
\hline 49 & $\mathrm{NA}$ & $\mathrm{NA}$ & 527 & 299 & 552 & NA & 43 & 15 & 43 & 41 \\
\hline 50 & NA & NA & 526 & 239 & 514 & $\mathrm{NA}$ & NA & NA & $\mathrm{NA}$ & NA \\
\hline 51 & NA & NA & 319 & 115 & 310 & NA & 13 & 8 & 29 & 17 \\
\hline 52 & $\mathrm{NA}$ & 2.5 & 72 & 106 & 84 & NA & 12 & 3 & 4 & 9 \\
\hline 53 & NA & $\mathrm{NA}$ & 111 & 175 & 134 & NA & 31 & 9 & 14 & 25 \\
\hline 54 & $\mathrm{NA}$ & $\mathrm{NA}$ & 236 & 345 & 299 & NA & $\mathrm{NA}$ & $\mathrm{NA}$ & $\mathrm{NA}$ & NA \\
\hline 55 & $\mathrm{NA}$ & $\mathrm{NA}$ & 318 & 27 & 1840 & $\mathrm{NA}$ & 12 & 4 & 34 & 7 \\
\hline 56 & NA & NA & 145 & 17 & 987 & NA & 6 & 6 & 19 & 5 \\
\hline 57 & NA & NA & 131 & 18 & 793 & NA & 3 & 4 & 10 & 2 \\
\hline 58 & NA & NA & 118 & 13 & 638 & NA & 4 & 4 & 13 & 4 \\
\hline 59 & $\mathbf{N A}$ & NA & 198 & 18 & 1190 & NA & 4 & 4 & 10 & 2 \\
\hline
\end{tabular}

Concentrations of all components are in micromolar units, except where indicated. Charge is omitted from all components except for Fe and S. LWC, liquid water content. DCP, direct current plasma. BDL, below detection limit. NA, not measured or not applicable. Read $3.3 E+16$ as $3.3 \times 10^{16}$.

* Fe for DPKBH analysis was fixed in the field [Garcia-Vargas et al., 1986; Zatar et al., 1989], and the measurements were carried out in the laboratory. All the Fe-ferrozine measurements were carried out in the field. 
Table 2: Changes of $\mathrm{Fe}$ (II) Concentrations in Cloudwater Samples Stored in Daylight and in the Dark as a Function of Time

\begin{tabular}{lrrcc}
\hline Sample & $\begin{array}{c}\text { Fe(II), } \\
\mu \mathrm{M}\end{array}$ & $\begin{array}{c}\text { Time Lapsed, } \\
\mathrm{min}\end{array}$ & Dark/light & $\begin{array}{c}\Delta \mathrm{Fe}(\mathrm{II}) / \Delta \mathrm{t}, \\
\mathrm{nM} / \mathrm{s}^{*}\end{array}$ \\
\hline San Pedro1 & 1.43 & 0 & light & - \\
San Pedro1 & 3.04 & 30 & light & 0.90 \\
San Pedro1 & 4.18 & 105 & dark & 0.25 \\
San Pedro1 & 5.14 & 165 & dark & 0.27 \\
San Pedro2 & 9.80 & 0 & light & - \\
San Pedro2 & 12.05 & -10 & light & - \\
San Pedro2 & 12.34 & -20 & dark & - \\
San Pedro2 & 12.23 & -30 & dark & - \\
San Pedro3 & 13.05 & 0 & light & - \\
San Pedro3 & 14.81 & -10 & light & - \\
San Pedro3 & 14.66 & -20 & dark & - \\
& & & & \\
\hline
\end{tabular}

* Change in [Fe(II)] and time lapsed since the last measurement.

etate/formate ratios ( $1: 1)$ than the data obtained from samples falling on the line with a lower slope (acetate/formate 0.5). Anthropogenic continental air masses appear to have higher acetate/formate ratios than air masses originating in remote environments [Keene and Galloway, 1984; Talbot et al., 1988; Gunz and Hoffmann, 1990]. Therefore the relationship between Fe and formate appears to be determined primarily by their common source region, although redox reactions cannot be ruled out. Samples correlated along the line with a steeper slope in Figure $2 a$ are indicative of air masses of anthropogenic origin, while those correlated along the line with a lower slope appear to be cleaner air masses. In contrast, it seems that redox reactions, rather than a common source, control the relationship between Fe and acetate. This is suggested by the fact that in the Los Angeles samples the correlation between acetate and $\mathrm{Fe}(\mathrm{II})$ (Figure $2 \mathrm{c}$ ) is much better than the correlation between acetate and $\mathrm{Fe}_{\mathrm{ac}}$ (Figure $2 d$ ) and between $\mathrm{Fe}$ (II) and formate. Acetate is formed either by the oxidation of aldehydes as catalyzed by $\mathrm{Fe}$ (III)/Fe(II) or it is depleted by direct electron transfer to Fe(III) species; or both processes may take place simulaneously. The Fe-catalyzed oxidation of aldehydes to produce carboxylic acids might provide an important source of these acids to the troposphere [Keene and Galloway, 1986].

The relationship between Fe and oxalate is different from the observed linear relationships between $\mathrm{Fe}$ (II) and both formate and acetate (Figure $3 a$ ). Although we have analyzed relatively few cloudwater samples with measurable Fe(II) and oxalate concentrations, it appears that there is no correlation between their concentrations (Table 1, Figure $3 a$ ). Therefore it is suggested that Fe and oxalate do not have common sources and that oxalate has no net effect on the formation of $\mathrm{Fe}$ (II). On the other hand, the oxalate (but neither formate nor acetate) concentrations in cloudwater samples show a positive correlation with peroxide (Figure $3 a$ ). These observations suggests that Fe may have had a primary source of emission to the atmosphere, while oxalate had a secondary source (photochemical production with peroxide) in the atmosphere [Zuo and Hoigné, 1992].

Results from our ongoing laboratory investigation on the photoreduction of aerated suspensions of goethite, hematite, amorphous Fe hydroxide, and ambient aerosol particles are
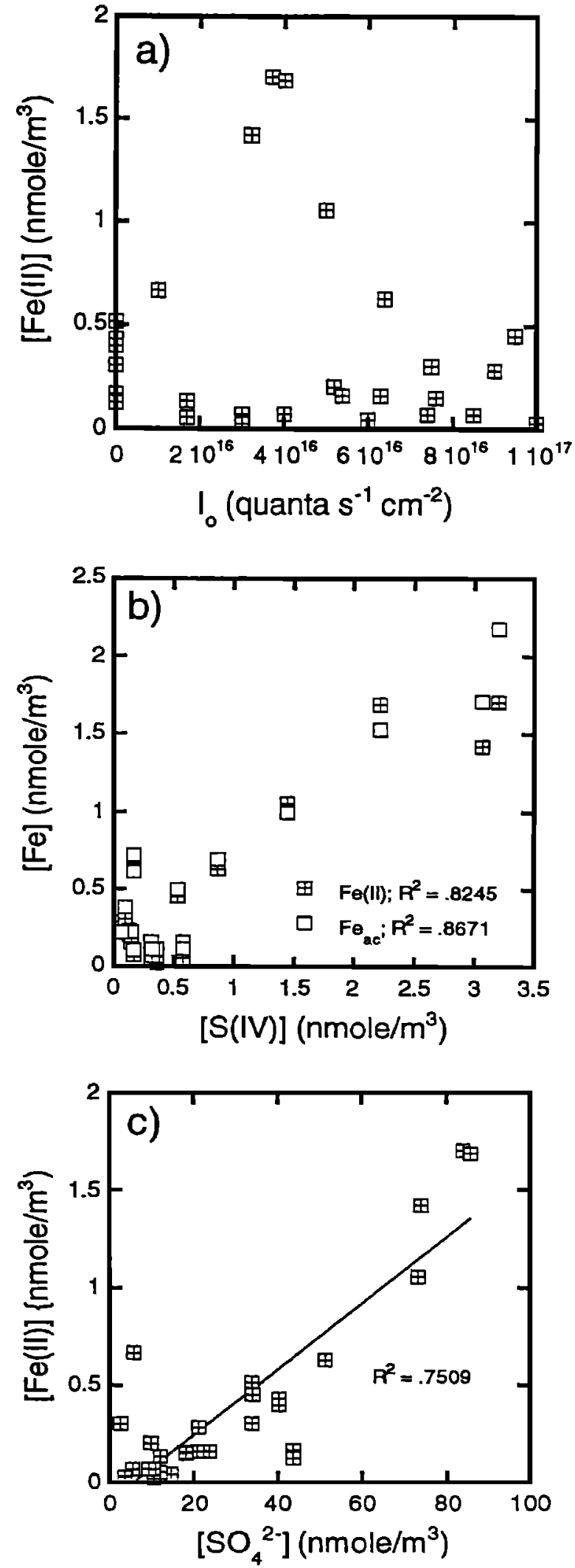

Fig. 1. Correlation between different parameters in cloudwater samples collected in Los Angeles. Concentrations were obtained by multiplying the measured concentration in a sample with the liquid water content of the cloud in order to obtain concentration per unit volume of air: (a) $\mathrm{Fe}$ (II) versus light, (b) $\mathrm{S}(\mathrm{IV})$ versus both $\mathrm{Fe}(\mathrm{II})$ and $\mathrm{Fe}_{\mathrm{ac}}$ (Fe(II)+Fe(III)) concentration in unfiltered acidified sample, and (c) Fe(II) versus sulfate. 

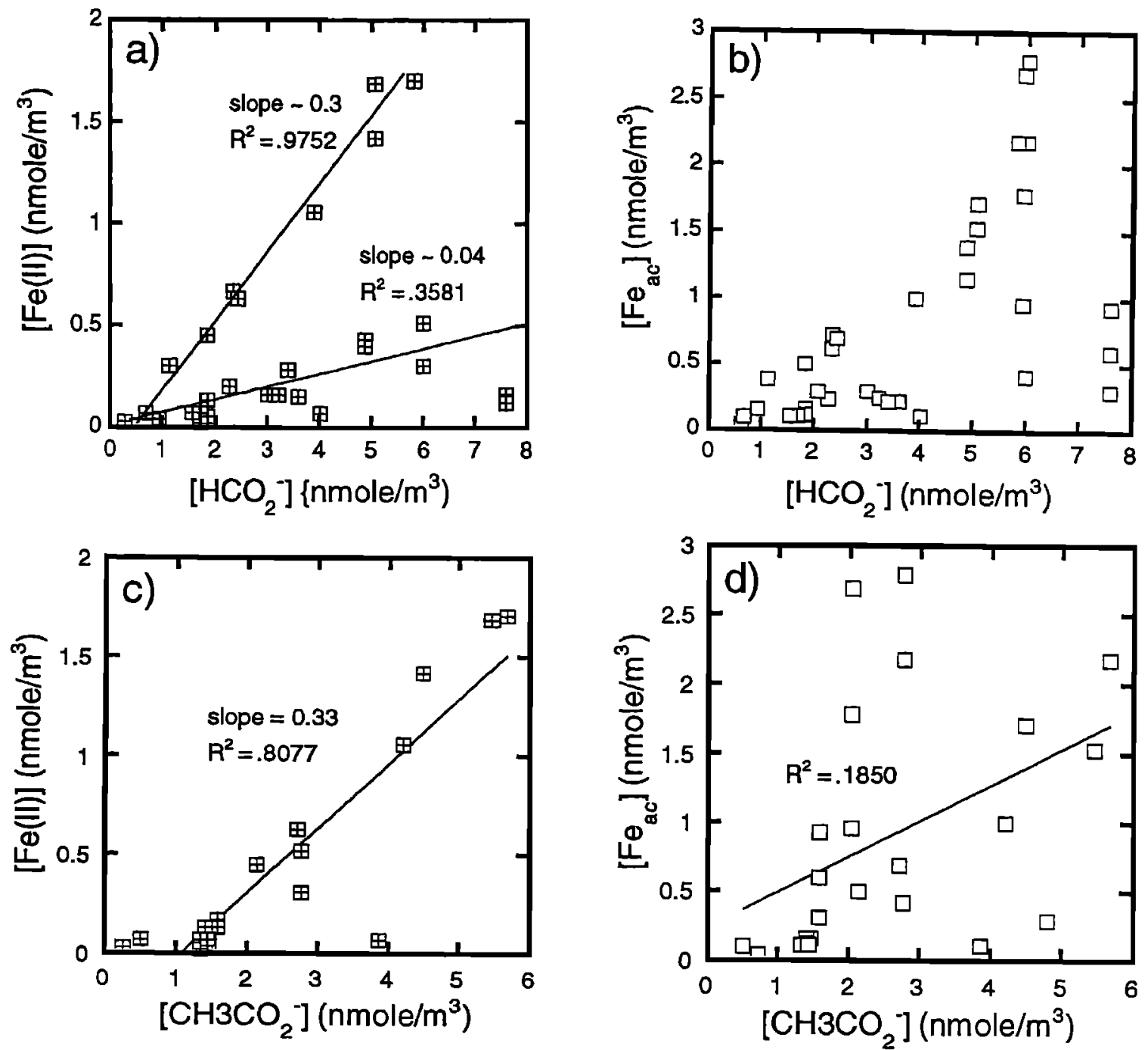

Fig. 2. The correlation between Fe and monocarboxylic acids in cloudwater samples collected in Los Angeles: (a) formate versus $\mathrm{Fe}(\mathrm{I})$, (b) formate versus $\mathrm{Fe}_{\mathrm{ac}},(c)$ acetate versus $\mathrm{Fe}(\mathrm{II})$, and $(d)$ acetate versus $\mathrm{Fe}_{\mathrm{ac}}$.

consistent with the results obtained in the field. In these laboratory experiments, no Fe(II) was detected in irradiated particle suspensions in the presence of oxalate, while at the same time large amounts of hydrogen peroxide were produced [Pehkonen et al., 1993]. When the same experiments were performed in the presence of formate and acetate, a significant fraction of Fe(III) was reduced to $\mathrm{Fe}$ (II) without the concomitant prodaction of hydrogen peroxide. Our observations are consistent with those of Wehrli et al. [1989] and Siffert and Sultzberger [1991]; they noted that Fe(II) is not produced in irradiated hematite suspensions in the presence of oxalate under oxic conditions. In these cases, adsorbed oxalate appears to be oxidized by dioxygen adsorbed on the surface of the Fe(III) oxyhydroxides to form hydrogen peroxide and carbon dioxide. The hydrogen peroxide in turn oxidizes $\mathrm{Fe}$ (II) released from the particle surface before it diffuses to the bulk solution. However, under anoxic conditions appreciable concentrations of $\mathrm{Fe}$ (II) were delected [Wehrli et al., 1989; Siffert and Sultzberger, 1991]. Based on these observations, there appears to be a unique photochemical behavior for oxalate in the presence of $\mathrm{Fe}(\mathrm{III})$ oxyhydroxide surfaces.
To assist in the interpretation of the aqueous phase speciation of $\mathrm{Fe}$, we have predicted, via thermodynamic calculation, the soluble Fe-containing species in the cloudwater samples. For these calculations we used the measured concentrations of components (including Fe(III) and Fe(II)) observed in an 1/2-hour period in a stratus cloud event that was 5 hours in duration (sample 47, Table 1; similar results were obtained with all the samples which contained appreciable concentrations of oxalate). The major $\mathrm{Fe}$ species were as follows: $\mathrm{Fe}\left(\mathrm{H}_{2} \mathrm{O}\right)_{2}\left(\mathrm{C}_{2} \mathrm{O}_{4}\right)_{2-}-\mathrm{Fe}\left(\mathrm{C}_{2} \mathrm{O}_{4}\right)_{33} 3$, $\mathrm{Fe}\left(\mathrm{H}_{2} \mathrm{O}\right)_{4}\left(\mathrm{C}_{2} \mathrm{O}_{4}\right)+$, and $\mathrm{Fe}\left(\mathrm{H}_{2} \mathrm{O}\right)_{6}+$ (Figures $3 b$ and $3 c$ ). Under the above assumptions (not considering any other organic ligands that might be present in solution) the three ferric iron species constitute more than $95 \%$ of the total dissolved Fe(III). This computation demonstrates the strong chemical interaction between oxalate and Fe(III). However, most of the cloudwater samples contained low levels of oxalate ( $<1-2$ micromole). Similar calculatioas done on these samples show that hydrolysis is the main ro action which controls the speciation of $\mathrm{Fe}$ (III). Among the $\mathrm{Fe}$ (III)-oxalate species, $\mathrm{Fe}\left(\mathrm{H}_{2} \mathrm{O}\right)_{2}\left(\mathrm{C}_{2} \mathrm{O}_{4}\right)_{2}$ - which is photochemically very reactive [Vincze and Papp, 1987] makes up 

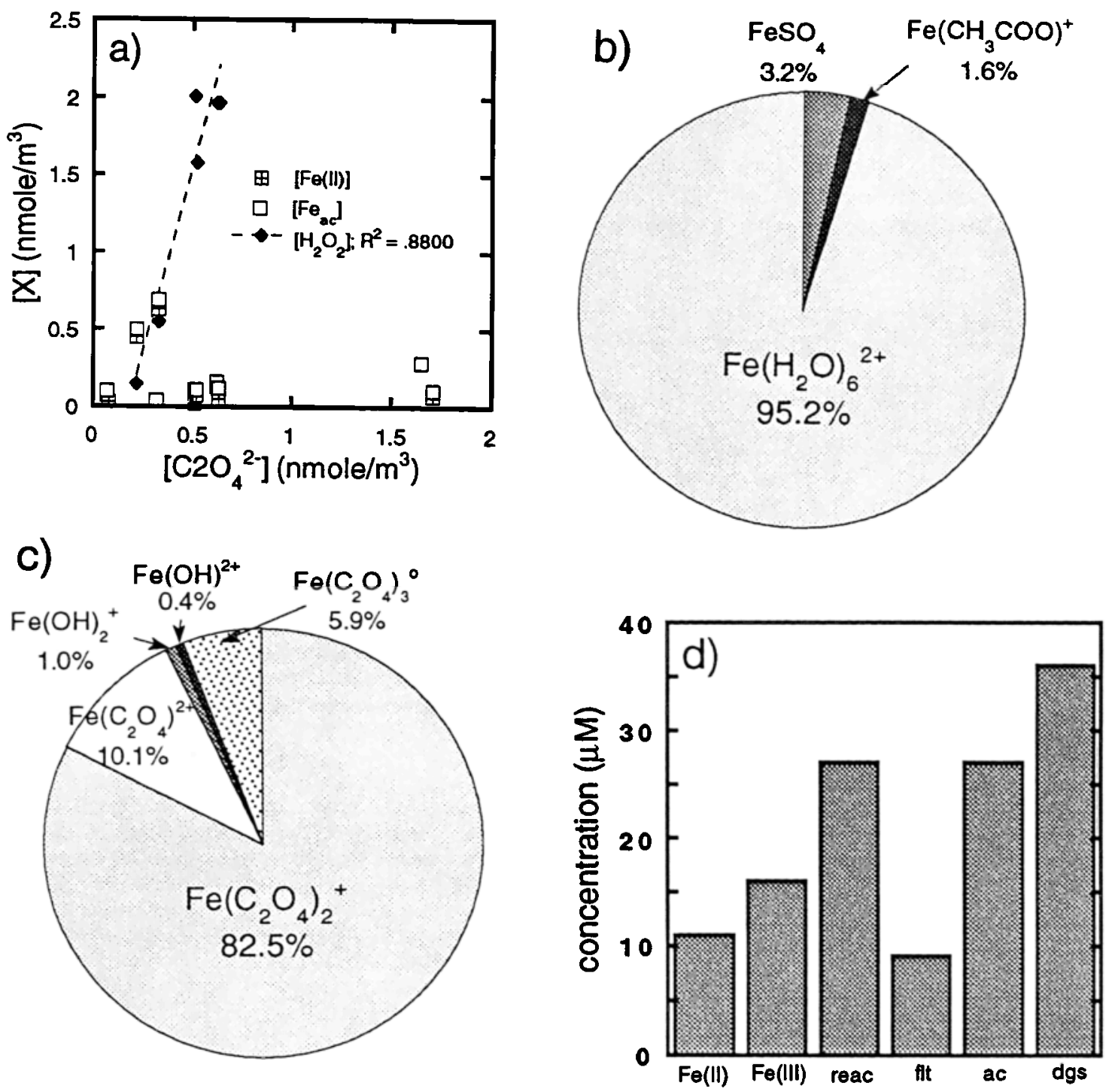

Fig. 3. (a) oxalate vs. $\mathrm{Fe}$ (II). Feac, and peroxides, (b) speciation calculation of $\mathrm{Fe}(\mathrm{II})$, and (c) speciation calculation of $\mathrm{Fe}(\mathrm{III})$. Both $(a)$ and $(b)$ were done on sample 47. Table 1. The measured concentrations are (micromole): $[\mathrm{Fe}(\mathrm{III})]=1.2 ;[\mathrm{Fe}(\mathrm{II})]=1.8 ;[$ sulfate $]=260 ;[$ (acetate+formate) $]=77 ;$ [oxalate $]=13 ;$ [hydrogen peroxide] $=41$; [formaldehyde] $=10 ; p H=3.4 ;(d)$ measured "dissolved" and particulate Fe in cloudwater sample. $\mathrm{Fe}$ (II), $\mathrm{Fe}$ (III) were measured spectrophotometrically in unfiltered sample aliquot, reac $=\mathrm{Fe}_{\mathrm{Teac}}$ the sum of the previous columns, flt $=\mathrm{Fe}_{\mathrm{flt}}$. (both oxidation states) measured by direct current plasma (DCP) in a filtered aliquot of the sample ( 0.025 micrometer Millipore filter) $; a c=F_{a c}$. (both oxidation states) measured by DCP in an aliquot of the sample that was acidified to $p H=1$ and then filtered; dgs $=F_{e_{d g s}}$, sum of $F_{e_{f l t}}$ and $F e$ (both oxidation states) measured by DCP in particles collected on the filter after they were digested with concentrated $\mathrm{HF}$ and $\mathrm{HNO}_{3}$.

$85 \%$ of the Fe(III) concentration. Oxalate reacts with hexaquo $\mathrm{Fe}$ (III) to form an inner-sphere complex which then undergoes a ligand-to-metal electron transfer (i.e., to produce $\mathrm{Fe}(\mathrm{II}) \mathrm{C}_{2} \mathrm{O}_{4}-$. [Zuo and Hoigné, 1992]). Fe(II), which is present mostly as $\mathrm{Fe}\left(\mathrm{H}_{2} \mathrm{O}\right)_{6} 2+$, is likely to be oxidized via one of the following pathways: (1) oxidation by dissolved oxygen (reasonably fast at $p \mathrm{H}$ above 5 [Stumm and Morgan, 1981; Millero et al., 1987]), (2) peroxides, and (3) ozone [Hoigné et al., 1985]. Of the three pathways it seems that in cloudwater samples of $p \mathrm{H}$ below 4.5 (i.e., all the samples except those collected in Bakersfield) Fe(II) reaction with peroxides to form $\mathrm{OH}$ radicals is the predominant pathway:

$$
\begin{aligned}
& \mathrm{Fe}^{2+}+\mathrm{H}_{2} \mathrm{O}_{2} \rightarrow \mathrm{Fe}^{3+}+\cdot \mathrm{OH}+\mathrm{OH}^{-} \\
& \left.\mathrm{FeOH}^{+}+\mathrm{H}_{2} \mathrm{O}_{2} \rightarrow \mathrm{Fe}(\mathrm{OH})\right)^{+}+\cdot \mathrm{OH}
\end{aligned}
$$

The species-dependent reactivity of $\mathrm{Fe}(\mathrm{II})$ toward hydrogen peroxide is seen clearly by comparison of $k_{6}(76 \mathrm{M}-1 \mathrm{~s}-1)$ [Walling, 1975] and $k_{7}(1.9 \times 106 \mathrm{M}-1 \mathrm{~s}-1)$ [Moffett and Zika, 1987]. Fe(II) also reacts with hydroperoxyl radical to form hydrogen peroxide,

$$
\begin{aligned}
& \mathrm{Fe}^{2+}+\mathrm{HO}_{2}+\mathrm{H}_{3} \mathrm{O}^{+} \rightarrow \\
& \mathrm{Fe}^{3+}+\mathrm{H}_{2} \mathrm{O}_{2}+\mathrm{H}_{2} \mathrm{O},
\end{aligned}
$$


and with peroxymonosulfate [Sato et al., 1984] to yield $\mathrm{Fe}(\mathrm{III})$ and $\mathrm{S}(\mathrm{V})$ as $\mathrm{SO}_{4}^{-}:$

$$
\mathrm{Fe}^{2+}+\mathrm{HSO}_{5}^{-} \rightarrow \mathrm{FeOH}^{2+}+\mathrm{SO}_{4}^{-}
$$

The samples collected in Bakersfield have the lowest content of $\mathrm{Fe}(\mathrm{II})$ although their $\mathrm{Fe}_{\mathrm{ac}}$ content is similar to other samples and they have relatively high concentrations of formate and acetate (Table 1). It is interesting to note that the Bakersfield samples have $p \mathrm{H}$ values above 6.4. At this $p \mathrm{H}$ range, oxidation of $\mathrm{Fe}$ (II) by oxygen (Haber-Weiss mechanism) is a rapid process operating in addition to the oxidation of $\mathrm{Fe}$ (II) by peroxides (which itself is faster at high $p \mathrm{H}$ ) to suppress the concentration of $\mathrm{Fe}(\mathrm{II})$ in solution. Another explanation for the low Fe(II) content of the Bakersfield samples is discussed in the next paragraph.

The similarity between the field results and the laboratory experiments on particle suspensions implies that photoassisted heterogeneous reactions are taking place within cloudwater droplets. The presence of surface-bound $\mathrm{Fe}$ is indicated by the results shown in Figure $3 d$. In this case, the concentration of reactive $\mathrm{Fe}\left(\mathrm{Fe}_{\mathrm{reac}}=[\mathrm{Fe}(\mathrm{II})]+\right.$ [Fe(III)]) was always found to be larger than filtered $\mathrm{Fe}, \mathrm{Fe}$ flt (i.e., $[\mathrm{Fe}(\mathrm{II})+\mathrm{Fe}$ (III)] $<0.025$ micrometer), and was either smaller or equal to $\left[\mathrm{Fe}_{\mathrm{ac}}\right] .\left[\mathrm{Fe}_{\mathrm{ac}}\right]$ is the sum of [Fe(II)] and [Fe(III)] measured as total $\mathrm{Fe}$ in an unfiltered sample at $p \mathrm{H}=1$. This latter value includes $\mathrm{Fe}$ that is dissolved, $\mathrm{Fe}$ released to solution from particle surfaces and $\mathrm{Fe}$ released to solution from the dissolution of oxyhydroxides at $p \mathrm{H} 1 . \mathrm{Fe}_{\mathrm{ac}}$ values in the samples were always lower than total $\mathrm{Fe}$ values ( $\mathrm{Fe}$ dgs, the concentration of $\mathrm{Fe}$ in filtrate plus filter digest; i.e., digested with concentrated HF and $\mathrm{HNO}_{3}$ ), suggesting that only a fraction of the total $\mathrm{Fe}$ in aerosols is likely to be released to cloudwater either by proton promoted or by reductive dissolution. $\mathrm{Fe}_{\mathrm{ac}} / \mathrm{Fe}_{\mathrm{dgs}}$ ratios varied largely from one sample to another, suggesting that the fraction of reactive $\mathrm{Fe}$ in aerosol samples was sensitive to the nature of the Fe-containing phases, which in turn were determined by the sources of $\mathrm{Fe}$ to the atmosphere. Such observations might explain the discrepancies between different authors regarding the fraction of reactive Fe in atmospheric aerosols [Zhuang et al., 1992; Zhu er al., 1993]. Based on selective leaching experiments (R. Siefert, California Institute of Technology, personal communication, 1992) it appears that approximately $5-10 \%$ of the total $\mathrm{Fe}$ in aerosols collected in Los Angeles was associated with natural and anthropogenic organic compounds via surface coatings and intergrowth. These organic compounds could serve as electron donors to reduce particulate Fe(III). Furthermore, the low $\mathrm{Fe}$ (II) content of the high $p \mathrm{H}$ samples from Bakersfield in spite of high formate and acetate concentrations is in accordance with the well-documented observations that both organic ligands and bisulfite are not likely to be adsorbed to Fe-rich phases at high $p \mathrm{H}$, and therefore heterogeneous electron transfer reactions are less likely to take place [Stumm and Morgan, 1981; Faust et al., 1989].

\section{CONCLUSIONS}

We suggest that the redox cycle of $\mathrm{Fe}$ in the near-shore continental troposphere involves both dissolved and aerosol surface species. Only a fraction of the total $\mathrm{Fe}$ measured in atmospheric aerosols is likely to participate in these redox reactions, and this fraction varies greatly from one sample to another. Photoreduction of Fe(III) in fog and cloudwater samples appears to be more significant than thermal reduction, although $\mathrm{Fe}$ (II) was also detected in the dark. Fe(III) reduction is determined primarily by oxidizable organic compounds, which undergo thermal and photoassisted oxidation by Fe(III) to yield carboxylic acids. The strong correlation observed between $\mathrm{Fe}(\mathrm{II})$ and organic matter, in the face of much weaker correlation between total $\mathrm{Fe}$ and organic matter, suggests chemical interdependency between $\mathrm{Fe}$ and some organic compounds (e.g., acetate) in fog and cloudwater samples. In addition, $\mathrm{Fe}$ (III)-organic compound reactions may provide an important source of carboxylic acids in the troposphere.

Acknowledgments. The authors wish to thank George Luther, Tom Church, Alan Veron, and the crew of RV Cape Henlopen, the College of Marine studies at the University of Delaware, Cliff Weinman and his staff at the FAA radar facility in San Pedro, Ted Murphy and Mel Dutton of the California State University, Bakersfield, and the staff of LA county fire department at Henninger Flats for their help in sample collection. The authors also wish to thank J.J. Morgan, B. Faust, and J. Winchester for their helpful comments. Support for this work was provided by a grant from the National Science Foundation (ATM 9015775); additional support for the work in Delaware Bay was provided by NSF grant OCE 8916804.

\section{REFERENCES}

Anderson, M. A., and F. M. M. Morel, The influence of aqueous iron chemistry on the uptake of iron by the coastal diatom Thalassira weissflogii, Limnol. Oceanogr., 27, 789-813, 1982.

Behra, P., and L. Sigg, Evidence for Redox Cycling of Iron in Atmospheric Water Droplets, Nature, 344, 419-421, 1990.

Carter, P., Spectrophotometric determination of serum iron at the submicrogram level with a new reagent (Ferrozine), Anal. Biochem., 40, 450-458, 1971.

Conklin, M. H., and M. R. Hoffmann, Metal ion-sulfur(IV) chemistry: Thermodynamics and kinetics of transient iron(III)-sulfur(IV) complexes, Environ. Sci. Technol., 22. 899-907, 1988.

Cunningham, K. M., M. C. Goldberg, and E. R. Weiner, The aqueous photolysis of ethylene glycol adsorbed on goethite, Photochem. Photobiol., 41, 409-416, 1985.

Daube, B. C. J., R. C. Flagan, and M. R. Hoffmann, Active cloudwater collector, US Pat. No. 4,697,462, 1987.

Dedik, A. N., P. Hoffmann, and J. Ensling, Chemical characterization of iron in atmospheric aerosols, Atmos. Environ., 26(A), 2545-2548, 1992.

Duce, R. A., The impact of atmspheric nitrogen, phosphorous and iron species on marine biological productivity, in The Role of Air-Sea Exchange in Geochemical Cycling, edited by P. Buat-Ménard, pp. 497-529, D. Reudel Publishing, D. Reidel, Norwell, Mass., 1986.

Faughnan, B., SURFEQL - An Interactive Code for Circulation of Chemical Equilibria in Aqueous Solution, W. M. Keck Laboratories, Calif. Inst. of Technol., Pasadena, 1981.

Faust, B. C., and M. R. Hoffmann, Photoinduced Reductive Dissolution of hematite by Bisulfite, Environ. Sci. Technol., 20, 943-948, 1986.

Faust, B. C., and J. Hoigne, Photolysis of Fe(III)-hydroxy complexes as sources of $\cdot \mathrm{OH}$ radicals in clouds, fog and rain, Atmos. Environ., 24, 79-89, 1990.

Faust, B. C., M. R. Hoffmann, and D. W. Bahnemann, Photoinduced oxidation of sulphur dioxide in aqueous suspen. sions of hematite, J. Phys. Chem., 93, 6371-6381, 1989.

Garcia-Vargas, M., M. Belizon, M. P. Hernandez-Artiga, C. 
Martinez, and J. A. Pcrez-Bustamante, Ligands with the "ONNN' group as chelating and preconcentrating substances of heavy metal ions: Aroylhydrazone derivatives from di-2pyridyl ketone, $A$. Spectros., 40, 1058-1062, 1986.

Gunz, D. W., and M. R. Hoffmann, Field investigations on the snow chemistry in central and southern California-II. Carbonyls and carboxylic acids, Atmos. Environ., 24(A), 1673-1684, 1990.

Hansen, L. D., D. Silberman, G. L. Fisher, and D. J. Eatough, Chemical speciation of clements in stack-collected, respirable-size coal fly ash, Environ. Sci. Technol., 18, 181186, 1984.

Hoffmann, M. R., and D. J. Jacob, Kinetics and mechanism of the catalytic oxidation of Ddissolved $\mathrm{SO} 2$ in atmospheric droplets: Free radical, polar and photoassisted pathways, in Acid Precipitation: $\mathrm{SO}_{2}, \mathrm{NO}, \mathrm{NO}_{x}$ Oxidation Mechanisnis: Atmospheric Considerations, edited by J. G. Calvert, pp. 101-172, Butterworth, Stoneham, Mass., 1984.

Hoigne, J., H. Bader, W. R. Haag, and F. Staehelin, Rate constants of reactions with ozone with organic and inorganic compounds in river water, III. Inorganic compounds and radicals, Water Res., 19, 993-1004, 1985.

Hong, H., and D. R. Kester, Redox State of Iron in the Offshore Waters of Peru, Lininol. Oceanogr., 31, 512-524, 1986.

Humphrey, R. E., M. H. Ward, and W. Hinze, Spectrophotometric determination of sulfite with 4,4'-dithiodipyridine and 5,5'-dithiobis-(2-nitrobenzoic acid), Anal. Chem., 42, 698702, 1970.

Jackson, G. A., and J. J. Morgan, Trace metal-chelator interactions and phytoplankton growth in seawater media: theoretical analysis and comparison with reported observations, Limnol. Oceanog., 230, 268-282, 1978.

Jacob, D. J., and M. R. Hoffmann, A dynamic model for the productiopn of $\mathrm{H}+, \mathrm{NO}_{3}-$, and $\mathrm{SO}_{4} 2-$ in urban fog, J. Geophys. Res., 88, 6611-6621, 1983.

Jacob, D. J., J. M. Waldman, J. W. Munger, and M. R. Hoffmann, The $\mathrm{H}_{2} \mathrm{SO}_{4}-\mathrm{HNO}_{3}-\mathrm{NH}_{3}$ system at high humidities in fogs: I. Spatial and temporal patterns in the San Joaquin Valley of California, J. Geophys. Res., 91, 1073-1088, 1986.

Kawamura, K., and I. R. Kaplan, Organic compounds in rainwater, in Organic Chemistry of the Atmosphere, edited by $\mathrm{L}$. D. Hansen, and D. J. Eatough, Pp. 233-284, CRC Press, Boca Raton, Fla., 1991.

Keene, W. C., and J. N. Galloway, Organic acidity in precipitation of North America, Atmos. Environ., 18, 2491-2497, 1984.

Keene, W., and J. N. Galloway, Considerations regarding sources for formic and acetic acids in the troposphere, Journal of Geophys. Res., 91, 14,466-14,474, 1986.

Kok, G. L., K. Thompson, and A. L. Lazrus, Derivatization technique for the determination of peroxides in precipitation, Anal. Chem., 58, 1192-1194, 1986.

Landing, W. M., and S. Westerlund, The solution chemistry of iron(II) in Framvaren Fjord, Marine Chem., 23, 329 - 343, 1988.

Martin, J. H., S. E. Fitzwater, and R. M. Gordon, Iron deficiency limits phytoplankton growth in the North-East Pacific subarctic, Nature, 321, 341-343, 1988.

Martin, J. H., R. M. Gordon, S. Fitzwater, and W. W. Broenkow, Vertex: Phytoplankton/iron studies in the Gulf of Alaska, Deep-Sea Res., 36, 649-680, 1989.

Martin, L. R., M. W. Hill, A. F. Tai, and T. W. Good, The iron catalyzed oxidation of sulfur(IV) in aqueous solution: Differing effects of organics at high and low $p \mathrm{II}, J$. Geophys. Res., 96, 3085-3097, 1991.

McKnight, D. M., B. A. Kimball, and K. E. Bencala, Iron photoreduction and oxidation in an acidic mountain stream, Science, $240,637-640,1988$.
Millero, F. J., S. Sotolongo, and M. Izaguirre, The oxidation kinetics of $\mathrm{Fe}(\mathrm{II})$ in seawater, Geochim. Cosmochin Acta, 51, 793-801, 1987.

Moffett, J. W., and R. G. Zika, Reaction kinetics of hydrogen paroxide with copper and iron in seawater, Environ. Sci. Technol., 21, 804-810, 1987.

Morales, C., Saharan Dust - Mobilization, Transport, Deposition, John Wiley, New York, 1979.

Morel, F. M. M., and M. J. J., A numerical method for computing equilibria in aqueous chemical systems, Environ. Sci. Technol., 6, 58-67, 1972.

Munger, J. W., The chemical composition of fogs and clouds in Southern California, Ph. D. thesis, Calif. Inst. of Technol., 1989.

Olson, T. M., and M. R. Hoffmann, Hydroxyalkylsulfonate formation: its role as a $S(I V)$ reservoir in atmospheric water droplets, Atmos. Environ., 23, 985-997, 1989.

Pandis, S. N., and J. H. Seinfeld, Mathematical modeling of acid deposition due to radiation fog, J. Geophys. Res., 94. 12911-12923, 1989.

Pandis, S. N., J. H. Seinfeld, and C. Pilinis, Haterogeneous sulfate production in an urban fog, Atmos. Environ., 26(A), 2509-2522, 1992.

Pehkonen, S. O., Y. Erel, and M. R. Hoffmann, Simultaneous spectrophotometric measurement of $\mathrm{Fe}$ (II) and $\mathrm{Fe}$ (III) in atmospheric water, Environ. Sci. Technol., 26, 1731-1736, 1992.

Pehkonen, S. O., R. Siefert, S. Webb, Y. Erel, and M. R. Hoffmann, Photoproduction of iron oxybydroxides in the presence of important atmospheric organic compounds, Environ. Sci. Technol., in press, 1993.

Sato, T., T. Gotto, T. Okabe, and F. Lawson, The oxidation of $\mathrm{Fe}(\mathrm{II})$-sulfate with sulfur dioxide and oxygen mixtures, $B$ ull. Chem. Soc. Jpn., 57, 2082-2086, 1984.

Siffert, C., and B. Sulzberger, Light-induced dissolution of hematite in the presence of oxalate: A case study, Langmuir, 7, 1627-1634, 1991.

Smith, R. V., and P. W. Erhardt, Nash determination for formaldehyde in the presence of bisulfite, Anal. Chem., 47, 2462-2464, 1975.

Stookey, L. L., Ferrozine: a new spectrophotometric reagent for iron, Anal. Chem., 42, 119-781, 1970.

Stumm, W., and J. J. Morgan. Aquatic Chemistry, John Wiley, New York, 1981.

Talbot, R. W., K. M. Beecher, R. C. Harriss, and W. R. Cofer, Atmospheric chemistry of formic and acetic acids at a midlatitude temperate site, J. Geophys. Res., 93, 1638-1652, 1988.

Vincze, L., and S. Papp, Individual quantum yields of $\mathrm{Fe}^{3+\mathrm{OX}} \mathrm{a}^{\mathrm{2}}$. $\mathrm{H}^{\mathrm{m}+}$ complexes in aqueous acidic solutions $\left(\mathrm{OX}^{2-}=\mathrm{C}_{2} \mathrm{O}_{4}{ }^{2-}\right.$, $\mathrm{n}=1, \mathrm{~m}=0,1), J$. Photochem., 36, 289-296, 1987.

Walling, C., Fenton's reagent revisited, Acc. Chent. Res., 8, 125-131, 1975.

Wehrli, B., B. Sulzberger, and W. Stumm, Redox processes catalyzed by hydrous oxide surfaces, Chem. Geol., 78, 167. $179,1989$.

Weschler, C. J., M. L. Mandich, and T. E. Graedel, Speciation, photosensitivity, and reactions of transition metal ions in atmospheric droplets, J. Geophys. Res., 91, 5189-5204, 1986.

Westall, J. C., Adsorption mechanisms in aquatic surface chemistry, in Aquatic Surface Chemristry, edited by W. Stumm, pp. 3-31, John Wiley, New York, 1987.

Zatar, N. A., A. Z. Abu-Zuhri, M. A. Al-Nuri, F. M. Mahmoud, and A. A. Abu-Obaid, Simultaneous spectrophotometric determination of iron(II) and iron(III) in mixtures using di-2pyridyl ketone benzoylhydrazone, Spec. Letters, 22, 1203 1214, 1989.

Zhu, X. R., J. M. Prospero, D. L. Savoie, F. J. Millero, R. G. 
Zika, and E. S. Saltzman, Photoreduction of iron(III) in marine mineral aerosol solutions, J. Geophys. Res., 98, 90399046, 1993.

Zhuang, G., Y. Zhen, and R. A. Duce, Link between iron and sulphur cycles suggested by detection of $\mathrm{Fe}(\mathrm{II})$ in remote marine aerosols, Nature, 355, 537-539. 1992.

Zhuang, G, The chemistry of iron in marine aerosols, Global Biogeochemical Cycles, 6, 161-173, 1992.

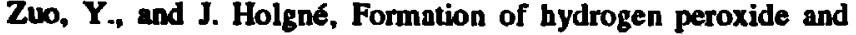
depletion of oxalic acid on atmospheric water by photolysis of iron(III)-oxalato compounds, Environ. Sci. Technol., 26, 1014-1022, 1992.

Y. Erel, S. O. Pehkonen and M. R. Hoffmann, W. M. Keck Laboratories of Environmental Engineering Science, California Institute of Technology, Pasadena, CA 91125.

(Received November 3, 1992; revised June 7, 1993; accepted June 8, 1993) 